\title{
Biological performance of grape varieties in Eastern Hungary
}

\author{
Rakonczás, $\mathbf{N}$. \\ University of Debrecen, Faculty of the Agricultural and Food Sciences and Environmental Management, \\ Institute of Horticulture 138. Böszörményi str., Debrecen, H-4032, Hungary
}

\begin{abstract}
Summary: Eurasian and interspecific grape varieties and candidates were inspected for biological productivity in the variety collection of the University of Debrecen at the Horticultural Experimental Station in Pallag. In this paper, data are reported on yields (kg/stock), cane production (kg/ stock) and use-up index of wood yield of each cultivar in the experimental years 2011-2012. From the range of Eurasian (Vitis vinifera L.) white wine-grape qualified cultivars and candidates 'Ezerfürtü', 'Generosa', 'Jubileum 75', 'Müller Thurgau', 'Zöld veltelíni', 'B-11', 'CSFT92', 'Pintes', from the range of Eurasian (Vitis vinifera L.) red wine-grape qualified cultivars and candidates 'Kármin', 'Pinot noir', 'Alicante Bouschet' and 'Rubintos' were highlighted. Promising data of interspecific 'Aletta', 'Csillám', 'Kunleány', 'Orpheus', 'Refrén', 'Taurus', 'Viktória gyöngye', 'Alföld 100', 'Reform', 'RF38/32', 'Toldi' white, and 'Dunagyöngye' 'Pannon frankos' red wine-grape cultivars and candidates were emphasized. Published data refer only to the biological performance of the cultivars. The thorough evaluation of varieties needs respect to data on resistance to fungal disease and climatic extremities, and other characteristics concerning production technology and oenological parameters.
\end{abstract}

Keywords: grape varieties, biological production, Y/N-ratio

\section{Introduction}

The total yearly wine production of our country is dynamically decreasing from the generally accepted 3 million hl, even down to 2,5 of 2011, and 1,8 of the vintage of 2012. The reason for this decrease could be searched in the background composed of many well-known world-economic tendencies, measures done by the leap forward in the name of environmentally sound plant protection technologies, global climate change, dynamic change of wine taste of the world and so on, which would be hard to tackle with even one by one (Hajdu-Borbásné, 2009; Sidlovits, 2008; Eperjesi, 2010).

The lower segment of the domestic wine market has lost its "appreciation", the pyramid of the domestic wine market is sensibly in collapse (Kopcsay, 2012). There became a huge niche emptied in the segment of the cheaper wines, which is "happily" filled up by Italian mass-wine producers (Kopcsay, 2012). However, it is important to say from the point of clear comprehension, that this quantity production does not directly mean lower wine quality. The theoretical bases are well circumscribed in works of Csepregi (1982) and Diófási (1985), followed by Lörincz \& Barócsi (2009).

The cultivation of varieties highlighted in this work or most of them - combined with adequate mechanization, production- and wine making technology, thanks to higher yields and the possibility of minimising costs of plant protection or reducing the highly damaging fungal diseases such as grape prudery mildew, mean a good opportunity for competitiveness to face the Italian imports (Hajdu, 2006; Szöke, 2006; Füzi \& Holb, 2007; Taksonyi et al., 2010; Holb \& Füzi, 2015).

An earlier paper deals with the oenological evaluation of some varieties of outstanding productivity (Rakonczás, 2011).

Most of the cultivars highlighted due to their outstanding data are lately ennobled and resistant. Since these varieties were created by the cross of Vitis vinifera and certain other traits of Vitis spp., these are called interspecific hybrids. Thanks to their character of being more or less resistant to fungal diseases on certain platforms these are referred to as "resistant" cultivars (Zanathy et al., 2005). The most novel distinction denominates them the PIWI-varieties (Pilzwiderstands-fähige Rebsorten) (Morandell, 2008).

According to the critique of the general comprehension a certain medicinal, or in case of red varieties a labrusca-taste is characteristic of wines of resistant wine-grape varieties. The formation of methanol by the process of downgrading pectin by pectin-methyl esterase represents a real weak point in the safety-evaluation of these varieties, and pushes back the spread of these vineyards.

By the adaptation of technological elements not detailed in this paper (such as superoxidization, the use of enzymes etc.) (Maya, 1994; Szöke, 2004; Kállay-Nyitrainé, 2004; Eperjesi, 2010; Kállay, 2010) many lately ennobled Hungarian resistant wine-grape varieties are published to be appropriate for quality wine production, and could 
be acquitted of negative critics (Phytowelt Gmbh, 2003, Nyitrainé et al., 2011; Hajdu, 2013).

According to our observation there are more, mostly white qualified grape varieties and candidates, which show higher level in productivity. In its evaluation it is vital to clarify, that higher yield in itself cannot be judged, only together with corresponding data on cane-, that is, the vegetative production. A respective and thorough scientific literature was lied down by Csepregi (1982). The figure (Y/N) is the quotient of the yield $(\mathrm{kg})$ and the cane production $(\mathrm{kg})$, and its normal interval is between 3 and 6. However, it can greatly deviate from this range, according to the vegetative or generative character of the variety, to applied phytotechnical practices and the condition of the plantation. It is also important to respect that the derived data hides the real total biological productivity, since $10 \mathrm{~kg} / 5 \mathrm{~kg}=2$ and $2 \mathrm{~kg} / 1 \mathrm{~kg}=2$ ! In this context Tomcsányi \& Német (1963) cit. Csepregi (1982) stated that variety is to be deemed valuable, of which the cane production between vintages does not show great variation, and in comparison to other cultivars, a considerably higher yield is harvested on the same level of cane production.

This work primarily aims to highlight varieties in our collection, which under the same conditions and production technology, show higher yields together with a considerably stable cane mass, thus the higher level of the yield does not result in the consequent decrease of vegetative production.

\section{Materials and methods}

The variety collection of the University of Debrecen was established in Pallag, on immune sandy soil, by $3 \mathrm{~m}$ between row and $1 \mathrm{~m}$ between stock spacing trained for single curtain stock form, with the use of European own rooted planting material, which is serious information from the point of view of the evaluation of data on productivity. Five stocks of each cultivar represent one experimental block.

Nutrition is carried out on the basis of the specific nutrient demand of the grape (Kozma, 1993) giving out $310 \mathrm{~kg} \mathrm{NPK}$ (effective material) manure on yearly bases in two phases. Farmyard manuring is carried out every four years. Dripping irrigation system is built out in the plantation.

The following data were collected: yield (kg/stock), cane production ( $\mathrm{kg} / \mathrm{stock}$ ), of which the $\mathrm{Y} / \mathrm{N}$-ratio, that is, the use-up index of wood yield is calculated ( $\mathrm{kg}$ yield $/ \mathrm{kg}$ cane).

\section{Results}

Table 1 does not list the whole variety collection, only those mostly interesting from the point of the present discussion. Besides outstanding cultivars, certain other varieties are also indicated, which are commonly cultivated.

Based on the yield data of 2011 and 2012 from the group of state qualified white European (Vitis vinifera) wine-grape cultivars 'Cserszegi füszeres', 'Ezerfürtü', 'Generosa', 'Jubileum 75', 'Müller Thurgau', 'Szürkebarát', 'Zenit' and
'Zöld veltelíni' should be mentioned. The listed varieties can be characterised by a normal average cane production. Only 'Ezerfürtü', 'Generosa' and 'Jubileum 75' show higher vegetative production besides the outstanding yields, resulting in a normal Y/N-ratio. In the case of 'Cserszegi füszeres', 'Müller Thurgau', 'Zenit' and 'Zöld veltelini', the higher generative production does not coincide with higher cane production.

From the group of European (Vitis vinifera) candidates 'B-11', 'CSFT-92', 'Kecskemét 13', 'Pintes' and 'Tarcal-4' are to be highlighted, of which 'CSFT-92' and 'Pintes' can be characterised with a simultaneous higher cane production. The vegetative production of 'Tarcal-4' is considerably low.

Looking at the data of red European (Vitis vinifera) wine-grape varieties, it is clear to see that in average, besides a considerably acceptable cane production - close to the general average - they show respectively lower yields resulting in a lower average $\mathrm{Y} / \mathrm{N}$-ratio.

Except for 'Pinot noir', 'Alicante Bouschet' and probably 'Zweigelt', 'Bíbor kadarka' and 'Merlot', it would be difficult to mention any of the red wine cultivars or candidates.

Table 2 shows data on interspecific wine-grape hybrids. It is basically good to see that there are several outstanding items in the group of white varieties and candidates, which results that the average yield production and Y/N-ratio are considerably higher than that of the qualified European (Vitis vinifera) varieties and intraspecific crossings.

In the group of qualified interspecific white wine-grape varieties 'Aletta', 'Csillám', 'Göcseji zamatos', 'Kunleány', 'Orpheus', 'Refrén', 'Taurus' and 'Viktória gyöngye' are to be mentioned for higher yields (close to $1.85 \mathrm{~kg} / \mathrm{m}^{2}$ ). It is interesting to notice that this higher yield is balanced by a cane production, which on its average is just the same as in the case of the European white wine-grape cultivars. However, there is a great difference between actual interspecific varieties. 'Aletta' shows higher, while 'Csillám', 'Kunleány', and 'Orpheus', show lower vegetative production.

In the group of white interspecific wine-grape candidates 'Alföld 100', 'Amadeus', 'Reform', 'RF38/32', and 'Toldi' are to be highlighted, of which, each variety is to be characterized by dominative vegetative production besides higher yields, thus their Y/N-ratio is considerably lower than that of the qualified interspecific white wine-grape varieties.

In the group of interspecific red wine-grape cultivars, there are only two items to be highlighted. These are 'Dunagyöngye' and 'Pannon frankos', both of which are to be characterised by a considerable vegetative overbalance, according to data.

\section{Conclusion}

Based on the data of 2011 and 2012 collected in the variety collection of the University of Debrecen, it is possible to nominate certain qualified varieties and candidates which would deserve more attention so that we can face winemarket problems detailed in the introduction. 
Table 1. Harvest data of white and red European (Vitis vinifera) wine-grape varieties and candidates (Pallag, 2011-2012)

\begin{tabular}{|c|c|c|c|c|c|c|c|c|c|c|}
\hline \multirow[b]{2}{*}{ Variety name } & \multicolumn{4}{|c|}{ Yields (kg/ stock) } & \multicolumn{3}{|c|}{$\begin{array}{l}\text { Use-up index of wood yield } \\
\text { (kg yield / } \mathrm{kg} \text { cane) }\end{array}$} & \multicolumn{3}{|c|}{$\begin{array}{c}\text { Wood yield } \\
\text { (kg cane / stock) }\end{array}$} \\
\hline & 2011 & 1012 & (yield/ stock) & (yield/m2) & 2011 & 2012 & Average & 2011 & 2012 & Average \\
\hline \multicolumn{11}{|c|}{ Eurasian white wine grape (Vitis vinif era L.) varieties and hybrids } \\
\hline \multicolumn{11}{|l|}{ Qualif ied varieties } \\
\hline Chardounay & 1,58 & 3,52 & 2,55 & 0,85 & 2,05 & 5,24 & 3,65 & 0,77 & 0,67 & $\mathbf{0 , 7 2}$ \\
\hline Cserszegif üszeres & 2,80 & 5,88 & 4,34 & 1,45 & 7,18 & 9,19 & 8,18 & 0,39 & 0,64 & 0,52 \\
\hline Ezerf ürtii & 2,03 & 8,85 & 5,44 & 1,81 & 3,03 & 7,14 & 5,09 & 0,67 & 0,83 & 0,75 \\
\hline Generosa & 2,40 & 7,84 & 5,12 & 1,71 & 2,29 & 8,97 & 5,63 & 1,05 & 0,87 & 0,96 \\
\hline Jubileum 75 & 4,74 & 6,92 & 5,83 & 1,94 & 6,81 & 7,08 & 6,94 & 0,70 & 0,98 & 0,84 \\
\hline Karát & 3,00 & 4,68 & 3,84 & 1,28 & 4,02 & 5,85 & 4,94 & 0,75 & 0,80 & $\mathbf{0 , 7 7}$ \\
\hline Királyleányka & 2,52 & 3,26 & 2,89 & 0,96 & 3,56 & 3,20 & 3,38 & 0,71 & 1,02 & 0,86 \\
\hline Korouna & 1,40 & 2,32 & 1,86 & 0,62 & 1,31 & 2,41 & 1,86 & 1,07 & 0,96 & 1,02 \\
\hline Müller Thurgan & 3,60 & 5,50 & 4,55 & 1,52 & 4,62 & 8,81 & 6,71 & 0,47 & 0,62 & 0,55 \\
\hline Olasz nizling & 1,62 & 2,36 & 1,99 & 0,66 & 5,79 & 6,59 & 6,19 & 0,28 & 0,36 & 0,32 \\
\hline Ottonel muskotály & 2,18 & 5,44 & 3,81 & 1,27 & 2,57 & 6,34 & 4,46 & 0,85 & 0,86 & 0,85 \\
\hline Raj nai rizling & 1,96 & 4,32 & 3,14 & 1,05 & 6,90 & 8,50 & 7,70 & 0,28 & 0,51 & $\mathbf{0 , 4 0}$ \\
\hline Rozália & 2,26 & 4,20 & 3,23 & 1,08 & 3,98 & 7,00 & 5,49 & 0,57 & 0,60 & 0,58 \\
\hline Szzürkebarát & & 5,92 & 5,92 & 1,97 & & 10,35 & 10,35 & 0,82 & 0,57 & $\mathbf{0 , 7 0}$ \\
\hline Tramini & 2,18 & 3,42 & 2,80 & 0,93 & 3,11 & 4,17 & 3,64 & 0,70 & 0,82 & 0,76 \\
\hline$Z_{\text {enit }}$ & & 5,94 & 5,94 & 1,98 & & 9,00 & 9,00 & 0,46 & 0,66 & $\mathbf{0 , 5 6}$ \\
\hline Zöld veltelini & 2,92 & 7,64 & 5,28 & 1,76 & 4,71 & 12,32 & 8,52 & 0,62 & 0,62 & $\mathbf{0 , 6 2}$ \\
\hline Average & 2,48 & 5,18 & 4,03 & 1,34 & 4,13 & 7,19 & 5,98 & $\mathbf{0 , 6 6}$ & $\mathbf{0 , 7 3}$ & 0,69 \\
\hline \multicolumn{11}{|l|}{ Candidates } \\
\hline$B-11$ & 3,56 & 9,42 & 6,49 & 2,16 & 7,04 & 16,47 & 11,75 & 0,51 & 0,57 & 0,54 \\
\hline \begin{tabular}{|l|} 
CSFT-92 \\
\end{tabular} & * & 11,74 & 11,74 & 3,91 & & 12,28 & 12,28 & 0,76 & 0,96 & 0,86 \\
\hline Kecskemét-13 & 2,34 & 6,58 & 4,46 & 1,49 & 4,68 & 9,91 & 7,29 & 0,50 & 0,66 & 0,58 \\
\hline Muscat Bouschet & 4,20 & 2,42 & 3,31 & 1,10 & 4,16 & 2,94 & 3,55 & 1,01 & 0,82 & 0,92 \\
\hline Nosztori rizling & 2,56 & 4,36 & 3,46 & 1,15 & 6,84 & 10,33 & 8,59 & 0,37 & 0,42 & 0,40 \\
\hline Pintes & 4,52 & 7,00 & 5,76 & 1,92 & 4,35 & 12,32 & 8,34 & 1,04 & 0,57 & $\mathbf{0 , 8 0}$ \\
\hline Tarcal-4 & 5,50 & 3,92 & 4,71 & 1,57 & 19,23 & 8,48 & 13,86 & 0,29 & 0,46 & 0,37 \\
\hline Average & 3,78 & 6,49 & 5,70 & 1,90 & 7,72 & 10,39 & 9,38 & 0,64 & 0,64 & 0,64 \\
\hline \multicolumn{11}{|c|}{ Eurasian red wine grape (Vitis vinif era L.) varieties and hybrids } \\
\hline \multicolumn{11}{|l|}{ Qualif ied varieties } \\
\hline Bibor kadarka & 2,76 & 3,56 & 3,16 & 1,05 & 3,63 & 4,40 & 4,01 & 0,76 & 0,81 & $\mathbf{0 , 7 9}$ \\
\hline Blauburger & 2,35 & 1,95 & 2,15 & 0,72 & 2,24 & 4,15 & 3,19 & 1,05 & 0,47 & $\mathbf{0 , 7 6}$ \\
\hline Cabenuet f rauc & 1,52 & 3,08 & 2,30 & 0,77 & 1,25 & 2,04 & 1,64 & 1,22 & 3,78 & 2,50 \\
\hline Cabentet sauviguon & 1,42 & 2,72 & 2,07 & 0,69 & 1,79 & 2,97 & 2,38 & 0,79 & 2,29 & 1,54 \\
\hline Donf elder & 1,62 & 4,27 & 2,94 & 0,98 & 4,38 & 7,49 & 5,93 & 0,37 & 0,86 & 0,61 \\
\hline Kadarka & & 1,14 & 1,14 & 0,38 & & 1,01 & 1,01 & 0,86 & 2,83 & 1,85 \\
\hline Kánmin & 3,48 & 2,08 & 2,78 & 0,93 & 7,77 & 3,87 & 5,82 & 0,45 & 1,35 & 0,90 \\
\hline Kékf rankos & $* * * * *$ & *****1,96 & & & & 3,07 & 3,07 & 0,38 & 1,60 & 0,99 \\
\hline Kék oportó & 2,60 & 1,48 & 2,04 & 0,68 & 7,51 & 3,32 & 5,42 & 0,35 & 0,45 & 0,40 \\
\hline Merlot & 2,18 & 4,40 & 3,29 & 1,10 & 4,20 & 4,85 & 4,53 & 0,41 & 0,54 & 0,48 \\
\hline Pinot noir & 3,12 & 7,30 & 5,21 & 1,74 & 10,06 & 13,95 & 12,01 & 0,52 & 0,52 & $\mathbf{0 , 5 2}$ \\
\hline Zweigelt & 2,26 & 4,78 & 3,52 & 1,17 & 5,00 & 9,68 & 7,34 & 0,45 & 0,49 & 0,47 \\
\hline Average & 2,33 & 3,34 & 2,78 & $\mathbf{0 , 9 3}$ & 4,78 & 5,06 & 4,70 & 0,63 & 1,33 & 0,98 \\
\hline \multicolumn{11}{|l|}{ Candidates } \\
\hline Alicante Bouschet & 8,50 & 5,25 & 6,88 & 2,29 & 6,51 & 5,74 & 6,13 & 0,52 & 0,37 & 0,44 \\
\hline Cs.V. 420 & 1,42 & 4,12 & 2,77 & 0,92 & 2,29 & 6,75 & 4,52 & 0,62 & 0,61 & 0,62 \\
\hline Cs.V. 525 & 2,24 & 3,14 & 2,69 & 0,90 & 3,02 & 3,79 & 3,41 & 0,74 & 0,83 & $\mathbf{0 , 7 9}$ \\
\hline Kunucvér & 1,30 & 1,94 & 1,62 & 0,54 & 1,51 & 1,78 & 1,65 & 0,69 & 1,09 & $\mathbf{0 , 8 9}$ \\
\hline Medoc noir & & 3,10 & 3,10 & 1,03 & & 11,65 & 11,65 & 0,24 & 0,27 & 0,25 \\
\hline Magyarf rankos & 1,43 & 4,05 & 2,74 & 0,91 & 2,46 & 7,11 & 4,78 & 0,58 & 0,57 & 0,58 \\
\hline Miklóstelep 7 & 1,10 & 1,92 & 1,51 & 0,50 & 1,41 & 3,18 & 2,30 & 0,62 & 0,60 & 0,61 \\
\hline Rubintos & 2,44 & 3,48 & 2,96 & 0,99 & 4,80 & 5,94 & 5,37 & 0,51 & 0,59 & $\mathbf{0 , 5 5}$ \\
\hline Average & 2,63 & 3,38 & 3,03 & 1,01 & 3,14 & 5,74 & 4,98 & 0,56 & 0,61 & 0,59 \\
\hline
\end{tabular}

* wasp damage (Paravespula germanica, Vespa crabro) $* * * * *$ drought injury

European (Vitis vinifera) white wine-grape varieties and crossings: 'Cserszegi füszeres', 'Ezerfürtü', 'Generosa', 'Jubileum 75', 'Müller Thurgau', 'Szürkebarát', 'Zenit', 'Zöld veltelíni', 'B-11', 'CSFT-92', 'Kecskemét 13', 'Pintes' and 'Tarcal-4'. European (Vitis vinifera) red wine-grape varieties: 'Pinot noir' and 'Alicante Bouschet'. Interspecific white wine-grape hybrids: 'Aletta', 'Csillám', 'Göcseji zamatos', 'Kunleány', 'Orpheus', 'Refrén', 'Taurus', 'Viktória gyöngye', 'Alföld 100', 'Amadeus', 'Reform', 'RF38/32' and 'Toldi'. Interspecific red wine-grape hybrids: 'Dunagyöngye' and 'Pannon frankos'. 
Table 2. Harvest data of interspecific white and red wine-grape hybrids (Pallag, 2011-2012)

\begin{tabular}{|c|c|c|c|c|c|c|c|c|c|c|}
\hline \multirow[b]{2}{*}{ Variety name } & \multicolumn{4}{|c|}{ Yields (kg/ stock) } & \multicolumn{3}{|c|}{$\begin{array}{l}\text { Use-up index of wood yield } \\
\text { (kg yield / kg cane) }\end{array}$} & \multicolumn{3}{|c|}{$\begin{array}{c}\text { Wood yield } \\
\text { (kg cane / stock) }\end{array}$} \\
\hline & 2011 & 1012 & (yield/ stock) & (yield/m2) & 2011 & 2012 & Average & 2011 & 2012 & Average \\
\hline \multicolumn{11}{|l|}{ Interspecif ic hybrids } \\
\hline \multicolumn{11}{|l|}{ White wine-grape varieties } \\
\hline \multicolumn{11}{|l|}{ Qualif ied varieties } \\
\hline Aletta & 5,60 & 8,76 & 7,18 & 2,39 & 8,62 & 8,28 & 8,45 & 0,65 & 1,06 & 0,85 \\
\hline Bianca & 1,20 & 6,52 & 3,86 & 1,29 & 1,46 & 7,80 & 4,63 & 0,82 & 0,84 & 0,83 \\
\hline \begin{tabular}{|l|} 
Csillám \\
\end{tabular} & 7,34 & 6,48 & 6,91 & 2,30 & 18,26 & 16,70 & 17,48 & 0,40 & 0,39 & 0,40 \\
\hline Göcsej izamatos & 3,14 & 6,78 & 4,96 & 1,65 & 5,00 & 10,46 & 7,73 & 0,63 & 0,65 & 0,64 \\
\hline \begin{tabular}{|l|} 
Kunleány \\
\end{tabular} & 2,88 & 6,26 & 4,57 & 1,52 & 4,73 & 15,05 & 9,89 & 0,49 & 0,42 & 0,45 \\
\hline \begin{tabular}{|l|} 
Odysseuss \\
\end{tabular} & $* * * *$ & 3,04 & 3,04 & 1,01 & & 7,04 & 7,04 & 0,56 & 0,43 & $\mathbf{0 , 5 0}$ \\
\hline Orpheus & 2,70 & 6,94 & 4,82 & 1,61 & 7,34 & 12,76 & 10,05 & 0,37 & 0,54 & 0,46 \\
\hline Ref réu & 6,18 & 6,92 & 6,55 & 2,18 & 8,68 & 10,78 & 9,73 & 0,71 & 0,64 & 0,68 \\
\hline Tautnis & 0,78 & 10,26 & $\mathbf{5 , 5 2}$ & 1,84 & 1,11 & 16,50 & 8,80 & 0,70 & 0,62 & 0,66 \\
\hline $\mid$ Viktónia gyöugye & 6,60 & 9,56 & 8,08 & 2,69 & 10,89 & 13,86 & 12,37 & 0,61 & 0,69 & 0,65 \\
\hline Average & 4,05 & 7,15 & 5,55 & 1,85 & 7,34 & 11,92 & 9,62 & 0,59 & 0,63 & 0,61 \\
\hline \multicolumn{11}{|l|}{ Candidates } \\
\hline Alf öld 100 & $* * * * 1,08$ & 4,56 & 4,56 & 1,52 & 1,01 & 5,23 & 3,12 & 1,07 & 0,87 & 0,97 \\
\hline Amadeus & $* * * *$ & 5,16 & 5,16 & 1,72 & & 8,84 & 8,84 & 0,80 & 0,58 & 0,69 \\
\hline Knistály & 1,78 & 6,16 & 3,97 & 1,32 & 1,32 & 5,31 & 3,31 & 1,35 & 1,16 & 1,26 \\
\hline Kunbarát & 3,76 & 3,36 & 3,56 & 1,19 & 5,75 & 4,93 & 5,34 & 0,65 & 0,68 & 0,67 \\
\hline Ref onm & $*$ & 6,52 & 6,52 & 2,17 & & 8,56 & 8,56 & 0,81 & 0,76 & 0,79 \\
\hline$R f-38 / 32$ & 4,40 & 9,76 & 7,08 & 2,36 & 5,08 & 11,84 & 8,46 & 0,87 & 0,82 & 0,85 \\
\hline Toldi & 9,30 & 10,70 & 10,00 & 3,33 & 10,15 & 14,62 & 12,39 & 0,92 & 0,73 & 0,82 \\
\hline \begin{tabular}{|l|} 
Vértes csillaga \\
\end{tabular} & 2,08 & 3,90 & 2,99 & 1,00 & 4,08 & 5,89 & 4,98 & 0,51 & 0,66 & 0,59 \\
\hline Average & 4,26 & 6,27 & 5,48 & 1,83 & 4,56 & 8,15 & 6,87 & 0,87 & 0,78 & 0,83 \\
\hline \multicolumn{11}{|l|}{ Red wine-grape varieties } \\
\hline \multicolumn{11}{|l|}{ Qualif ied varieties } \\
\hline Duna gyöngye & 3,14 & 8,60 & 5,87 & 1,96 & 5,79 & 26,38 & 16,09 & 0,54 & 0,33 & 0,43 \\
\hline Korai bíbor & & 2,94 & 2,94 & 0,98 & & 5,10 & 5,10 & 0,54 & 0,58 & 0,56 \\
\hline \begin{tabular}{|l|} 
Mediuna \\
\end{tabular} & 2,21 & 3,78 & 3,00 & 1,00 & 6,83 & 5,66 & 6,24 & 0,45 & 0,67 & 0,56 \\
\hline Pannon f rankos & **** 1,76 & 5,38 & 5,38 & 1,79 & 5,57 & 7,75 & 6,66 & 0,32 & 0,69 & 0,51 \\
\hline Tutrán & 2,00 & 4,94 & 3,47 & 1,16 & 3,41 & 6,77 & 5,09 & 0,59 & 0,73 & 0,66 \\
\hline Average & 2,45 & 5,13 & 4,13 & 1,38 & 5,40 & 10,33 & 7,84 & $\mathbf{0 , 4 9}$ & 0,60 & 0,54 \\
\hline \multicolumn{11}{|l|}{ Candidates } \\
\hline Regent & 1,10 & 2,03 & 1,57 & 0,52 & 2,23 & 3,96 & 3,09 & 0,82 & 0,51 & 0,67 \\
\hline Tizian & 1,80 & 4,94 & 3,37 & 1,12 & 2,95 & 6,38 & 4,67 & 0,61 & 0,77 & 0,69 \\
\hline Average & 1,45 & 3,49 & 2,47 & 0,82 & 2,59 & 5,17 & 3,88 & 0,72 & 0,64 & 0,68 \\
\hline
\end{tabular}

* wasp damage (Paravespula germanica, Vespa crabro)

**** late spring frost damage

\section{References}

Csepregi P. (1982): A szőlő metszése, fitotechnikai műveletei. Mezőgazdasági Kiadó

Csepregi P., Zilai J. (1988): Szőlőfajta-ismeret és -használat. Mezőgazdasági Kiadó, Budapest. 508.

Diófási L. (1985): A minőségi borszőlőtermesztés tudományos alapjai. Mezőgazdasági Kiadó, Budapest. 259.

Eperjesi I. (2010): Borászati technológia. Mezőgazda Kiadó, Budapest. 313.

Füzi I, Holb IJ (2007): A szőlőt fertőző lisztharmatgomba telelő alakjainak járványtani szerepe a szekszárdi borvidéken Növényvédelem, 43 (6): 237-245.

Hajdu E. (2006): Idevaló szőlőfajták, Biokultúra, 17 (5): 13-15. p. Hajdu E., Borbásné S.É. (2009): Abiotikus stresszhatások a szőlő életterében. Agroinform Kiadó, Budapest. 227.

Hajdu E. (2013): Magyar szőlőfajták, Alany-, csemege-, és borszőlő fajták. Mezőgazda Kiadó, Budapest. 464.
Holb IJ, Füzi I (2015): Monitoring of ascospore density of Erysiphe necator in the air in relation ot weather factors and powdery mildew development. In: Abtrtact book of International Workshop on Fungal Grape Diseases (IWFDG). p. 16.

Kállay M. (2010): Borászati kémia. Mezőgazda Kiadó, Budapest. 206. p.

Kopcsay L. (2012): Zsugorodó ágazat, omladozó borpiramis. Borászati füzetek, (5): 22-23.

Kozma P. (1993): A szőlő és termesztése. A szőlő szaporitása és termesztéstechnológiája. Akadémiai Kiadó, Budapest. 403.

Lörincz A., Barócsi Z. szerk. (2010): A szőlő metszése és zöldmunkái. Mezőgazda Kiadó, Budapest. 306.

Kállay M., Nyitrainé S.D. (2004): A hiperoxidáció hatása különféle eredetű borok érzékszervi tulajdonságaira. Borászati füzetek, (1): 9-11.

Maya E. (1994): A borkészítés speciális feladatai az interspecifikus fehér szőlőfajták körében. Borászati füzetek, 2 (18-19): 5-7.

Morandell, W. (2008): PIWI-Südtirol stellt sich vor. Obstbau Weinbau, (3): 89-91. 
Nyitrainé S.D., Leskó A., Kállay M.(2011): Rezisztens szőlőfajták színanyag-összetételének vizsgálata. Kutatás, Borászati füzetek. 4-8.

Phytowelt Gmbh, (2003): Study on the use of varieties of interspecific vines. Final report. 227.

Rakonczás N. (2011): Mostoha gyermekeink, Rezisztens szőlőfajták. Bor és Piac, (5-6): 14-17.

Sidlovits D. (2008): Az új borpiaci szabályozás eredetvédelemre vonatkozó előírásai. Borászati füzetek, (3): 36-41.

Szőke L. (2004): Bioszőlő, biobor. Mezőgazda Kiadó, Budapest. 193.
Szőke L. (2006): Az ökológiai szőlészet és borászat Magyarországon. Biokultúra, 17 (5): 6-7.

Taksonyi P, Tarczal E, Maráczi K, Holb I, Kocsis L (2010): Powdery mildew infection dependent on weather factors in vineyards near Keszthely in 2008. International Journal of Horticultural Science, 16 (2): 55-59.

Tomcsányi P., Németh L. (1963): A szőlőtermés elemzése és növekedése. Nemesített növényfajtákkal végzett országos fajtakísérletek eredményei. 417-948.

Zanathy G., Lőrincz A., Bényei F., Fazekas I. (2005): A régi direkttermőktől a génmódosított szőlőkig. A rezisztencianemesítés vázlatos története. Borászati füzetek. 1: 1-15. 